\title{
Functional Tests of Leg Muscle Strength and Power in Adults With Cystic Fibrosis
}

\author{
Emily Sheppard, Kerrie Chang, Jackie Cotton, Sonia Gashgarian, Danny Slack, Kenneth Wu, \\ Anna Michalski, Pat Fox, Anne L Stephenson, and Sunita Mathur
}

\begin{abstract}
BACKGROUND: Muscle weakness is an important systemic consequence in adults with cystic fibrosis, but it can be challenging to evaluate clinically. This study examined the validity of lowerextremity functional tests to assess quadriceps muscle strength and muscle power. METHODS: The subjects underwent 4 functional tests: 30-s sit-to-stand test, stair-climb power test, vertical jump height, and triple hop distance. Quadriceps muscle strength and power were tested by using a dynamometer (the accepted standard). Quadriceps strength was measured from 5 maximum voluntary isometric contractions to obtain peak torque. Quadriceps power was evaluated from the peak power and peak velocity attained during isotonic contractions of the quadriceps at a preset load of $20 \%$ of the peak torque. Pearson correlations were used to determine associations between functional tests and accepted measures of quadriceps strength and power. RESULTS: Fifteen adults with cystic fibrosis ( 9 males; mean \pm SD age, $32 \pm 13$ y; mean \pm SD FEV $_{1} \%$ predicted, $73 \pm 19$ ) completed the study. The stair-climb power test had the strongest correlations with peak torque $(r=0.84, P<.001)$ and power $(\mathrm{r}=0.65, P=.009)$. Vertical jump height was moderately correlated with quadriceps strength $(\mathrm{r}=0.62$, $P=.014)$ and quadriceps peak power $(\mathrm{r}=0.51, P=.048)$. Similarly, triple hop distance had moderate correlations with quadriceps strength $(\mathrm{r}=0.78, P=.001)$ and peak power $(\mathrm{r}=0.57, P=.026)$. The sit-to-stand test was only associated with quadriceps strength $(\mathrm{r}=0.55, P=.034)$. CONCLUSIONS: Functional tests can be applied clinically to measure leg muscle strength and power, with the stair-climb power test having the strongest associations with the standard measures. The utility of using functional tests to evaluate longitudinal changes in muscle function and its association with clinical outcomes should be examined in cystic fibrosis. Key words: cystic fibrosis; skeletal muscle; muscle strength; muscle power; quadriceps. [Respir Care 2019;64(1):40-47. (C) 2019 Daedalus Enterprises]
\end{abstract}

\section{Introduction}

Cystic fibrosis is the most common genetic disease in Caucasians that causes premature death, with the incidence ranging from $\sim 1: 2,200$ to 1:3,500 live births in North America and western Europe. ${ }^{1}$ With medical advances, the longevity of people living with cystic fibrosis has markedly improved. Based on registry data from Can-

Ms Sheppard, Ms Chang, Ms Cotton, Ms Gashgarian, Mr Slack, Mr Wu, Ms Michalski, and Dr Mathur are affiliated with the Department of Physical Therapy, University of Toronto, Toronto, Ontario, Canada. Mr Wu and Dr Stephenson are affiliated with the Adult Cystic Fibrosis Centre, St Michael's Hospital, Toronto, Ontario, Canada. Ms Fox is affiliated with the Musculoskeletal Health and Outcomes Research, St. Michael's Hospital, Toronto, Ontario, Canada.

Financial support was provided by Physiotherapy Foundation of Canada - BE Schnurr Research Grant. ada, the United States, and the United Kingdom, the estimated median age of survival is 40 years; the projected trend shows that those born in 2000 will have a median age of $\geq 50 \mathrm{y} .{ }^{2}$ With aging, the development of secondary

\footnotetext{
The study was conducted at the Muscle Function and Performance Laboratory, Department of Physical Therapy, University of Toronto, Toronto, Ontario, Canada.

$\mathrm{Mr} \mathrm{Wu}$ presented, in part, the results of this study at the North American Cystic Fibrosis Conference, held October 2015, in Phoenix, Arizona.

The authors have disclosed no conflicts of interest.

Correspondence: Sunita Mathur, Department of Physical Therapy, University of Toronto, 160-500 University Ave, Toronto, ON M5G 1V7 Canada. E-mail: sunita.mathur@utoronto.ca.
}

DOI: $10.4187 /$ respcare. 06224 


\section{Muscle Strength and Power in Adults With CF}

musculoskeletal complications, for example, skeletal muscle dysfunction, has become an important concern in this population. Many factors, such as loss of muscle mass, poor nutritional status, systemic inflammation, corticosteroid use, and low physical activity levels, may contribute to the development of muscle dysfunction in adults with cystic fibrosis. ${ }^{3}$ Muscle weakness of the quadriceps and hamstrings ( $\sim 60-80 \%$ of healthy controls) has been reported in a number of studies of adults with cystic fibrosis and associated with muscle atrophy. ${ }^{4-6}$ Muscle strength may also decline further during an acute pulmonary exacerbation. ${ }^{7}$ In relation to clinical outcomes, quadriceps strength is associated with the 6-min walk distance, ${ }^{6}$ which is a strong predictor of mortality in adults with cystic fibrosis. ${ }^{8}$ Therefore, the evaluation of muscle strength is clinically relevant to monitor the decline in physical function and muscle mass that could be addressed through exercise, nutrition, and pharmaceutical therapies.

Computerized dynamometers are the accepted standard tool for evaluating muscle performance. In studies of adults with cystic fibrosis, computerized dynamometers, for example, Cybex (CSMi, Stoughton, Massachusetts), have been used to characterize isometric or isokinetic torque, $, 5,6$ which reflects strength or the tension-generating capacity of the muscle. Dynamometers can also be used to evaluate muscle power (the ability of the muscle to generate highvelocity, forceful movements) and muscle endurance/fatigability (the ability of the muscle to do repeated work). Although the computerized dynamometer is the accepted standard tool for evaluating muscle performance, this device is not commonly used in the clinical setting due to their high cost, large space requirements, and technical expertise required for testing. ${ }^{9}$

As an alternative to strength testing, functional tests of lower-extremity muscle strength and/or muscle power can be more easily applied because they require minimal space, time, and equipment. Timed sit-to-stand tests (eg, $30 \mathrm{~s}$ or 1 min) and the Timed Up and Go test are commonly used in older adults and people with chronic conditions, for example, COPD, and show correlations with quadriceps strength. ${ }^{10,11}$ The stair-climb power test, which was developed for older adults as a functional measure of lower-extremity muscle power, ${ }^{12}$ has also been used in COPD. ${ }^{13}$ Because adults with cystic fibrosis generally span a younger age range than individuals with COPD, tests of lower extremity function that are more challenging, for example, the vertical jump test, ${ }^{14}$ may be more applicable to this population. Currently, the validity of functional tests for the assessment of quadriceps muscle strength and power has not been established in cystic fibrosis. The objective of this study was to determine the criterionrelated validity of functional tests in adults with cystic fibrosis by examining the association between laboratory-based measures of quadriceps muscle strength and power by using

\section{QUICK LOOK}

\section{Current knowledge:}

Quadriceps muscle strength is reduced in adults with cystic fibrosis compared with age-matched healthy adults. The evaluation of muscle strength in the literature has been limited to measurements of isometric and isokinetic peak torque by using laboratory-based equipment, (ie, computerized dynamometry), which may not be feasible to conduct in the clinical setting.

\section{What this paper contributes to our knowledge:}

Clinical tests of lower-extremity function were correlated with quadriceps muscle strength and muscle power, as measured on a computerized dynamometer. More challenging tests, for example, the stair-climb power test, may be more useful than the sit-to-stand test because it can address a wider range of functional abilities and has moderate-to-high associations with both muscle strength and power.

computerized dynamometry with clinical tests of lower-extremity muscle function.

\section{Methods}

A prospective, observational study of adults with cystic fibrosis was conducted from January 2015 to April 2016. Eligible subjects were English-speaking adults ( $\geq 18$ y old), with a confirmed diagnosis of cystic fibrosis by sweat chloride testing and/or genotyping. Exclusion criteria were pulmonary exacerbation that required oral or intravenous antibiotics in the past month, lung transplantation, pregnancy, colonization of Burkholderia cepacia complex, neuromuscular disease, or musculoskeletal pain. The study participants were screened for inclusion at the out-patient cystic fibrosis clinic, and those individuals who were eligible and interested in participating in the study were contacted for a study visit. Ethical approval was obtained from the hospital (REB 14372) and university (REB 31152) research ethics boards. All the participants provided written informed consent.

Testing was conducted in a single visit at a university laboratory and included quadriceps strength and power tests on the dynamometer (System 4 Pro, Biodex Medical Systems, Shirley, New York), and 4 functional tests: 30-s sit-tostand test, stair-climb power test, vertical jump test, and triple hop distance. There was a 5-min break between the Biodex dynamometer and the functional tests. Adverse events during testing were recorded (eg, trips or falls, muscle strain and/or pull, or pain). Age, sex, and dominant lower extremity were recorded at the beginning of the testing session, and height 


\section{Muscle Strength and Power in Adults With CF}

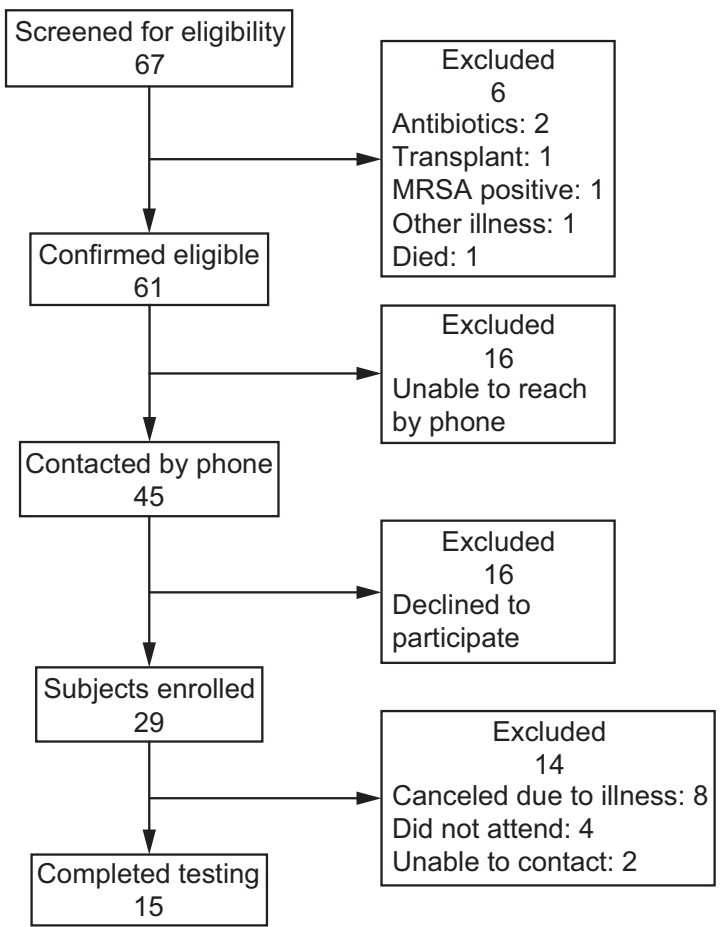

Fig. 1. Flow chart. MRSA $=$ Methicillan-resistant Staphylococcus aureus.

and weight were measured on a standing scale with shoes removed. Additional clinical characteristics were obtained from the participant's medical chart (age of diagnosis, cystic fibrosis genetic mutation, diagnosis of cystic fibrosis-related diseases (cystic fibrosis-related diabetes and cystic fibrosisrelated liver disease, osteoporosis or osteopenia), results of the most recent pulmonary function test $\left(\mathrm{FEV}_{1}\right.$ and $\mathrm{FVC}, \%$ predicted), ${ }^{15}$ bacterial status within the past year, and use of intravenous and/or oral glucocorticoid in the past 12 months.

\section{Muscle Strength and Power Testing on a Dynamometer}

Muscle strength (torque-generating capacity) and power (product of torque and velocity) of the knee extensors (quadriceps) were evaluated by using the Biodex dynamometer. Raw signals for torque, position, and velocity were sampled at $100 \mathrm{~Hz}$ and collected by using Acqknowledge version 4.1.1 (Biopac Systems, Goleta, California) for offline processing. Isometric peak torque (muscle strength) of the knee extensors was tested at $60^{\circ}$ of knee flexion. ${ }^{16}$ A practice trial was followed by 5 maximal voluntary trials with 1-min rest breaks. The subjects were instructed to push as hard and fast as possible, and verbal encouragement was provided throughout the trial. The average of the 3 highest values within 10\% was used for analysis. Isotonic muscle power was evaluated with the load set to $\sim 25-30 \%$ of the isometric peak torque mea-
Table 1. Participant Characteristics

\begin{tabular}{|c|c|}
\hline Characteristic & Results \\
\hline Age, mean $\pm \mathrm{SD}$ (range) y & $32 \pm 13(18-63)$ \\
\hline Males/females, $n$ & $9 / 6$ \\
\hline Height, mean $\pm \mathrm{SD} \mathrm{cm}$ & $162 \pm 15$ \\
\hline Weight, mean \pm SD kg & $66.3 \pm 15.1$ \\
\hline Body mass index, mean $\pm \mathrm{SD} \mathrm{kg} / \mathrm{m}^{2}$ & $25.9 \pm 9.7$ \\
\hline \multicolumn{2}{|l|}{ Pulmonary function tests } \\
\hline $\mathrm{FEV}_{1} \%$ predicted, mean $\pm \mathrm{SD}(\text { range })^{*}$ & $73 \pm 19(39-109)$ \\
\hline FVC $\%$ predicted, mean $\pm \mathrm{SD}$ (range) & $91 \pm 17(60-112)$ \\
\hline Inhaled steroids in the past year, $n(\%)$ & $12(80)$ \\
\hline Oral steroid use in past year, $n(\%)$ & $6(40)$ \\
\hline \multicolumn{2}{|l|}{ Comorbidities, $n(\%)$} \\
\hline Cystic fibrosis-related diabetes & $2(13)$ \\
\hline Pancreatic insufficient & $13(87)$ \\
\hline Cystic fibrosis-related liver disease & $1(7)$ \\
\hline Osteopenia $\dagger$ & $7(47)$ \\
\hline Osteoporosis $\ddagger$ & $1(7)$ \\
\hline \multicolumn{2}{|l|}{ Bacterial status, $n(\%)$} \\
\hline Pseudomonas aeruginosa & $13(87)$ \\
\hline Staphylococcus aureus & $13(87)$ \\
\hline Aspergillus & $11(73)$ \\
\hline Stenotrophomonas maltophilia & $7(47)$ \\
\hline Atypical mycobacteria & $2(13)$ \\
\hline Methicillin-resistant Staphylococcus aureus & $1(7)$ \\
\hline \multicolumn{2}{|l|}{ Genotype, $n(\%)$} \\
\hline Homozygous $\Delta \mathrm{F} 508$ & $5(33)$ \\
\hline Heterozygous $\Delta \mathrm{F} 508$ & $9(60)$ \\
\hline Other & $1(7)$ \\
\hline Age of diagnosis $\leq 2 \mathrm{y}$ & $9(60)$ \\
\hline \multicolumn{2}{|c|}{$\begin{array}{l}\text { * Predicted values for lung function were based on Global Lung Function Initiative (from } \\
\text { Reference } 14 \text { ). } \\
\dagger \text { Defined as a T score between }-2.5 \text { and }-1.0 \text {. } \\
\ddagger \text { Defined as a T score } \leq-2.5 \text {. }\end{array}$} \\
\hline
\end{tabular}

Table 2. Summary of Biodex Measures of Knee Extensor Strength and Power and Lower-Extremity Functional Tests

\begin{tabular}{lc}
\hline \hline \multicolumn{1}{c}{ Measure } & Results \\
\hline $\begin{array}{l}\text { Biodex dynamometer (accepted standard tool), } \\
\text { mean } \pm \text { SD }\end{array}$ \\
Knee extensor isometric peak torque, Nm \\
Peak isotonic velocity, degrees/s \\
Peak isotonic power, W \\
Functional tests, mean \pm SD \\
30-s sit-to-stand test, no. repetitions \\
Stair-climb power test, W \\
Triple hop distance, $\mathrm{cm}$ \\
$\quad$ Vertical jump test, cm & $422 \pm 48 \pm 18.4$ \\
\end{tabular}

sured in the previous test. ${ }^{17}$ The range of motion was set from $100^{\circ}$ of knee flexion to full extension. ${ }^{17} \mathrm{~A}$ familiarization set of 3 repetitions was followed by 2 sets of 10 maximal repetitions, with a 2 -min rest between sets. The mean 

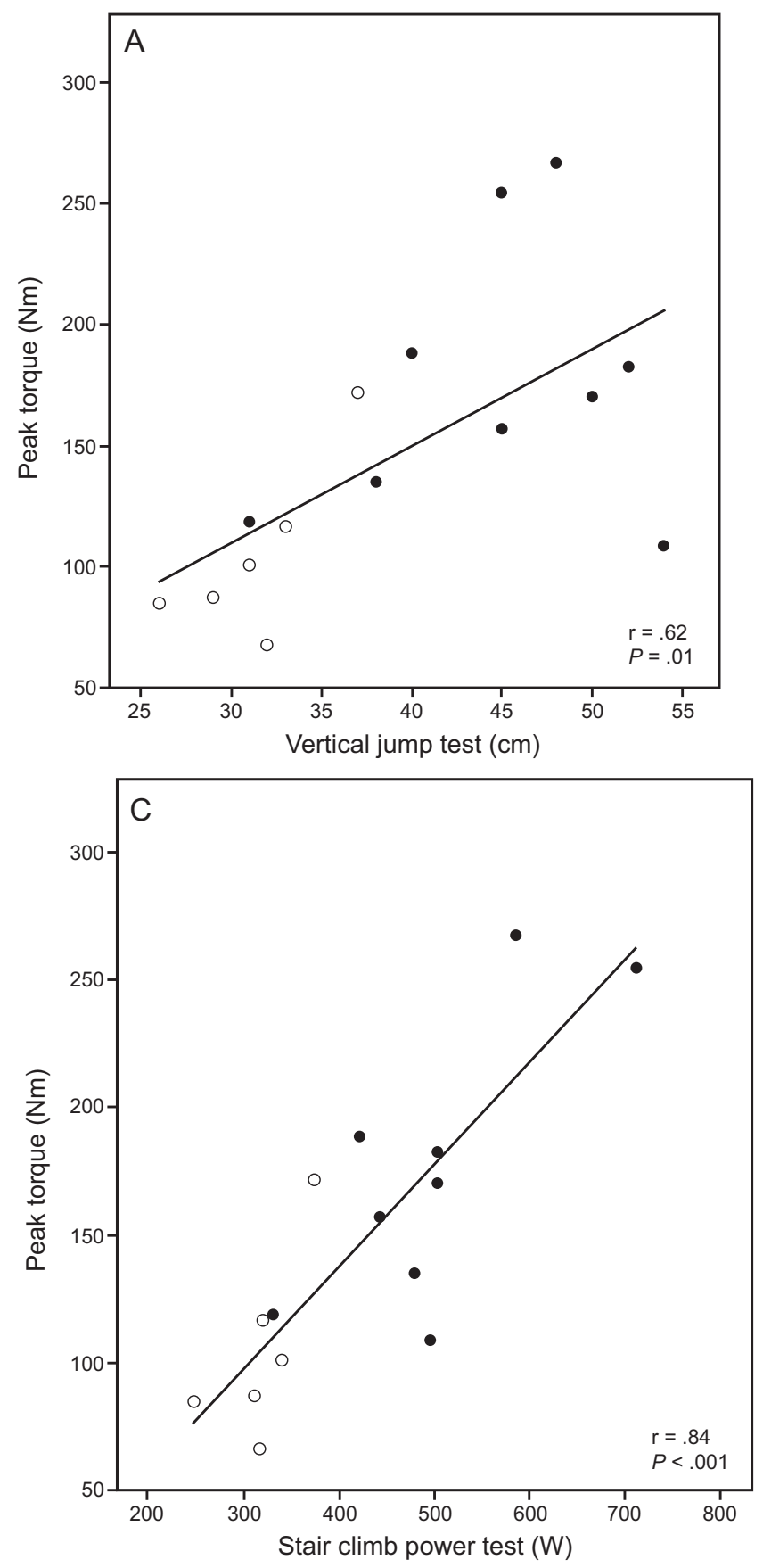

Fig. 2. Scatter plots of quadriceps peak torque versus functional tests.

of peak power (watts) and peak velocity (degrees/s) from the middle 6 contractions (excluding the first 2 and last 2 contractions from each set) were calculated; the highest value between the 2 sets was used for analysis.

\section{Lower-Extremity Functional Tests}

The participants performed 4 functional tests in random order, with 3-5 min between tests to reduce the influence of
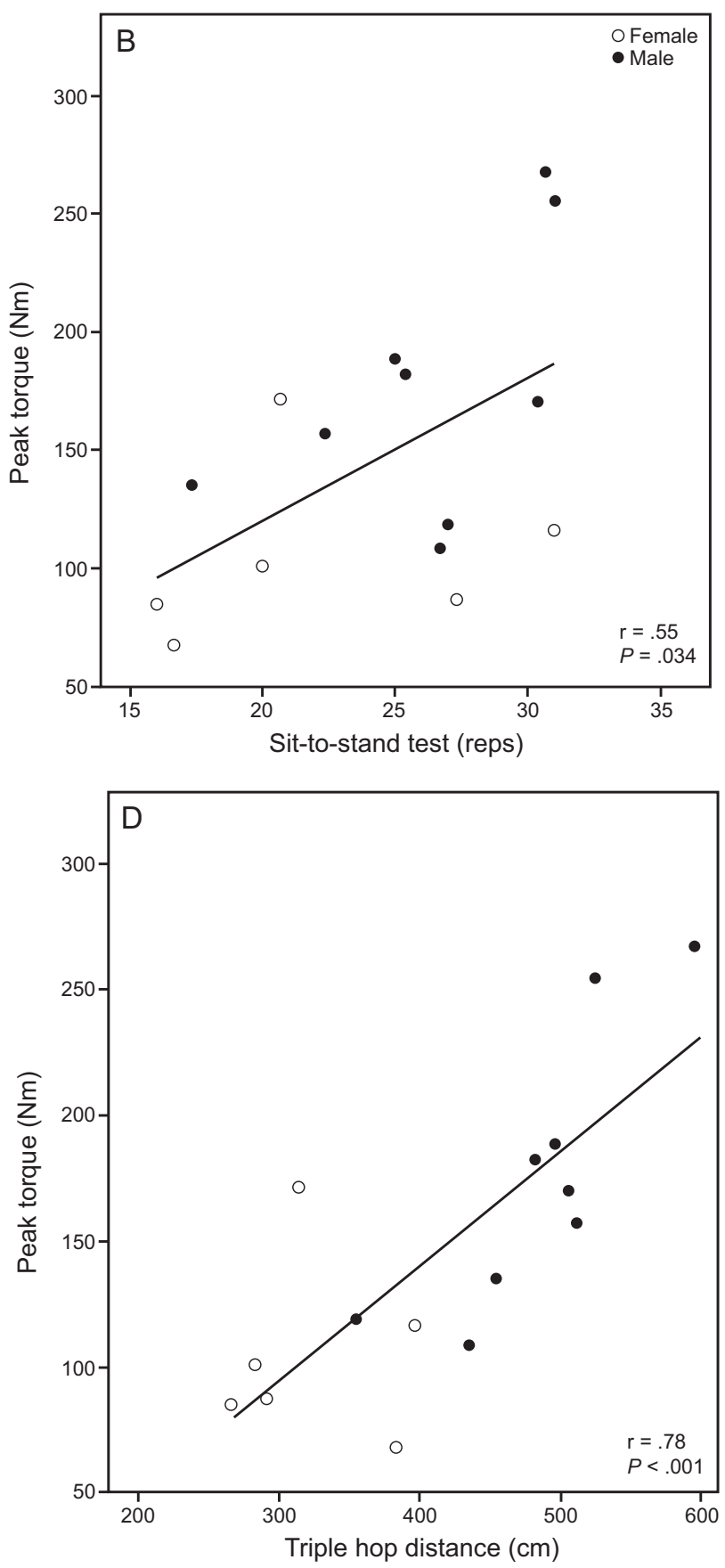

fatigue on performance. The participants observed the investigator (ES, KC, JC, DS, SG, and AM) demonstrate each test, which was followed by a practice trial. Three trials for each test were then performed, and the average score was used for analysis. The 30-s sit-to-stand test was conducted by using a standard $(46 \mathrm{~cm})$ height chair, with the subject seated in the middle of the chair and feet on the ground. The participant was instructed to stand from a seated position as many times as possible in $30 \mathrm{~s}$; the total number of full repetitions 

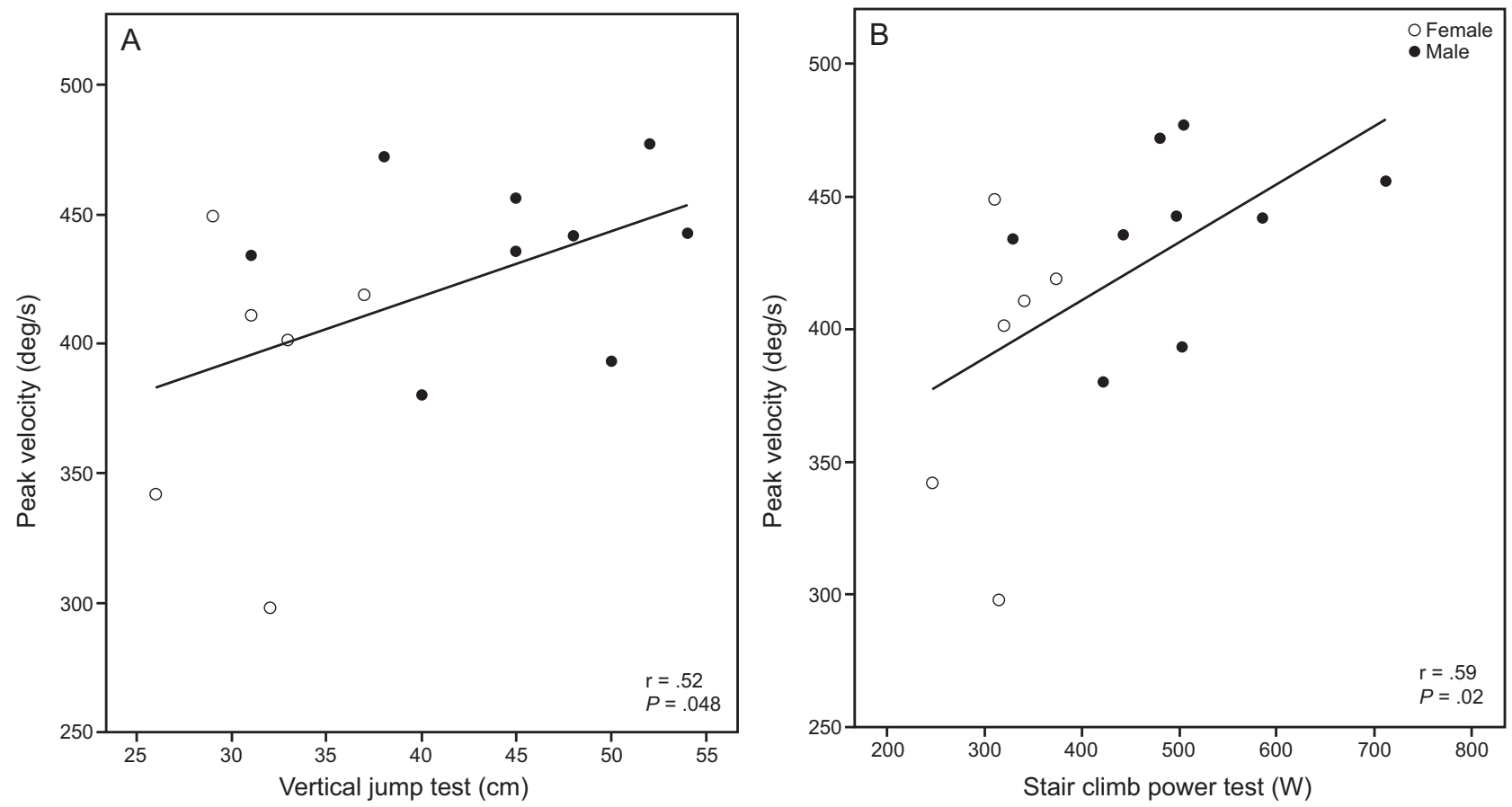

Fig. 3. Scatter plots of quadriceps peak velocity versus functional tests.

was recorded. One full repetition began and ended with the participant seated. ${ }^{18}$ For the stair-climb power test, the participants were asked to climb 10 stairs as fast as possible and the time was recorded in seconds. ${ }^{12}$ The timing began on the word "go" and ended when the participant had both feet on the top step. Leg muscle power (in watts) was calculated by using the equation by Bean et al. ${ }^{12}$

For the vertical jump test, the participant stood next to the wall with his or her dominant arm extended. The top of the middle finger was marked on the wall with chalk. The participant was then asked to jump as high as possible from a standing position with the arm stretched vertically and mark the wall at the top of the jump. The participant was permitted to bend his or her knees and trunk to initiate the jump, and arm swing was also permitted. ${ }^{14}$ The vertical jump height was measured as the difference between the standing reach height and the height of jump (in $\mathrm{cm}$ ). For the triple hop distance, a measuring tape was placed along the floor. The participant started at one end of the tape and was asked to hop 3 times consecutively on his or her dominant leg, trying to cover as much distance as possible. The distance hopped was recorded in centimeters. ${ }^{19}$

\section{Statistical Analysis}

Data were analyzed by using SPSS version 22.0 (SPSS, Chicago, Illinois). The Shapiro-Wilk test was used to determine normality of the variables, and all were normally distributed. Demographic and clinical variables were summa- rized by using mean $\pm \mathrm{SD}$ or frequencies (percentages). Pearson correlation coefficients were used to assess the relationships of knee extensor strength and power with each functional test, and were classified as low $(0.26-0.49)$, moderate (0.5-0.69), high (0.7-0.89), or very high $(0.9-1.0){ }^{20}$

\section{Results}

Sixty-seven adults with cystic fibrosis were screened for eligibility from the cystic fibrosis out-patient clinic: 61 were eligible, 29 agreed to participate, and 15 completed the study. The most common reason for not completing the study was illness (Fig. 1). The final sample included 9 men and 6 women, with a mean \pm SD age of $32 \pm 13$ (range, 18-63) y. Clinical characteristics of the sample are shown in Table 1. There were no adverse events during testing.

Knee extensor strength (isometric peak torque), power (peak power and peak velocity from isotonic contractions), and results of the functional tests are summarized in Table 2. Knee extensor strength showed moderate-to-high correlations with all functional tests and had the highest correlation with the stair-climb power test $(\mathrm{r}=0.84, P<.001)$ (Fig. 2). Knee extensor isotonic power measures showed moderate correlations with vertical jump height (peak power: $\mathrm{r}=0.51, P=$ .048 ; peak velocity: $\mathrm{r}=0.52, P=.048)$ and with stair-climb power (peak power: $\mathrm{r}=0.65, P=.009$; peak velocity: $\mathrm{r}=$ $0.59, P=.002$ ) (Figs. 3 and 4 ), and peak power also had a moderate correlation with the triple hop distance $(\mathrm{r}=0.57$, $P=.03$ ) (Fig. 4). There were no significant correlations 

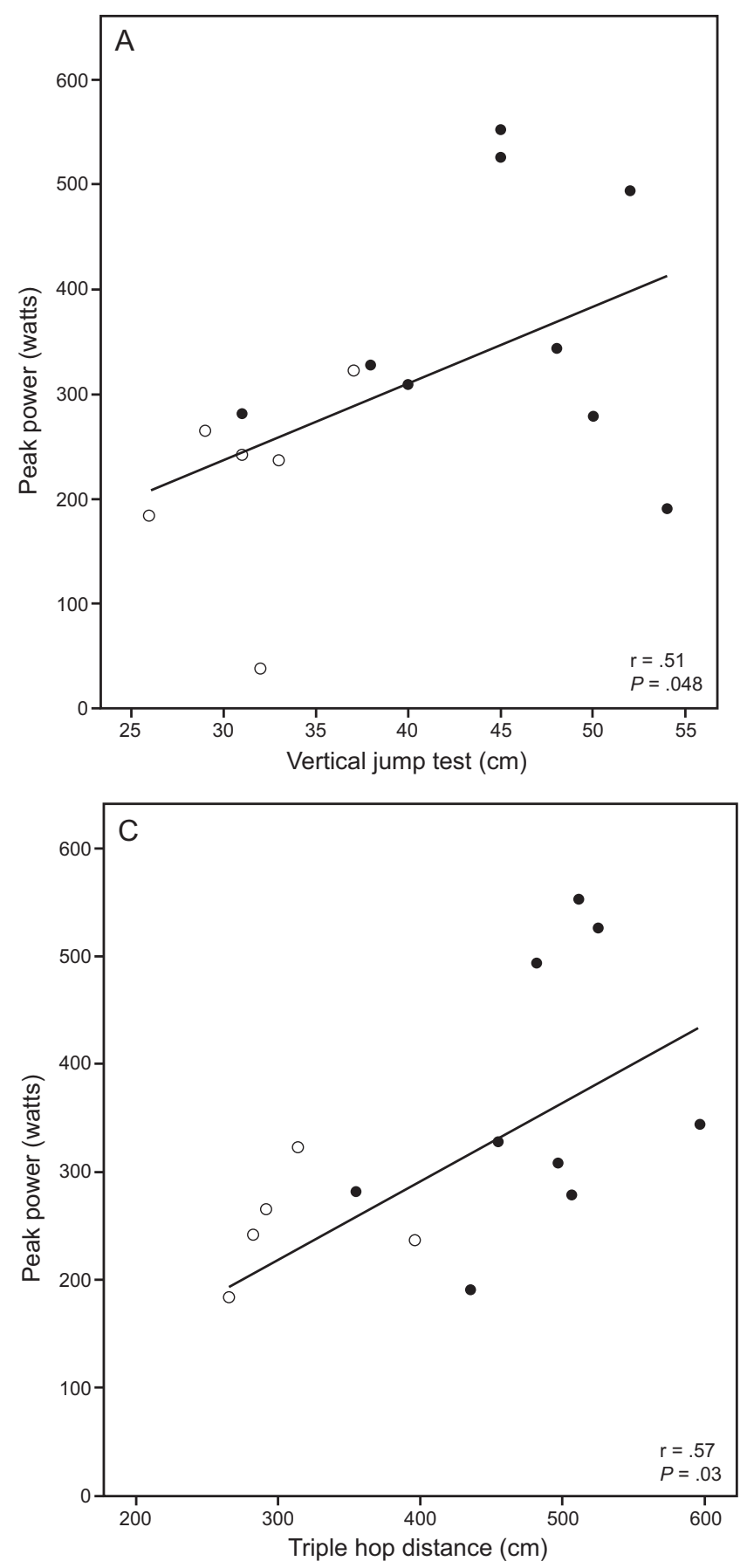

Fig. 4. Scatter plots of quadriceps peak power versus functional tests.

between peak velocity $(\mathrm{r}=0.45, P=.09)$ or peak power ( $\mathrm{r}=0.34, P=.22$ ) with the 30 -s sit-to-stand test or peak velocity with the triple hop distance $(\mathrm{r}=0.40, P=.14)$.

\section{Discussion}

This study examined the validity of lower-extremity functional tests to evaluate muscle strength and power by

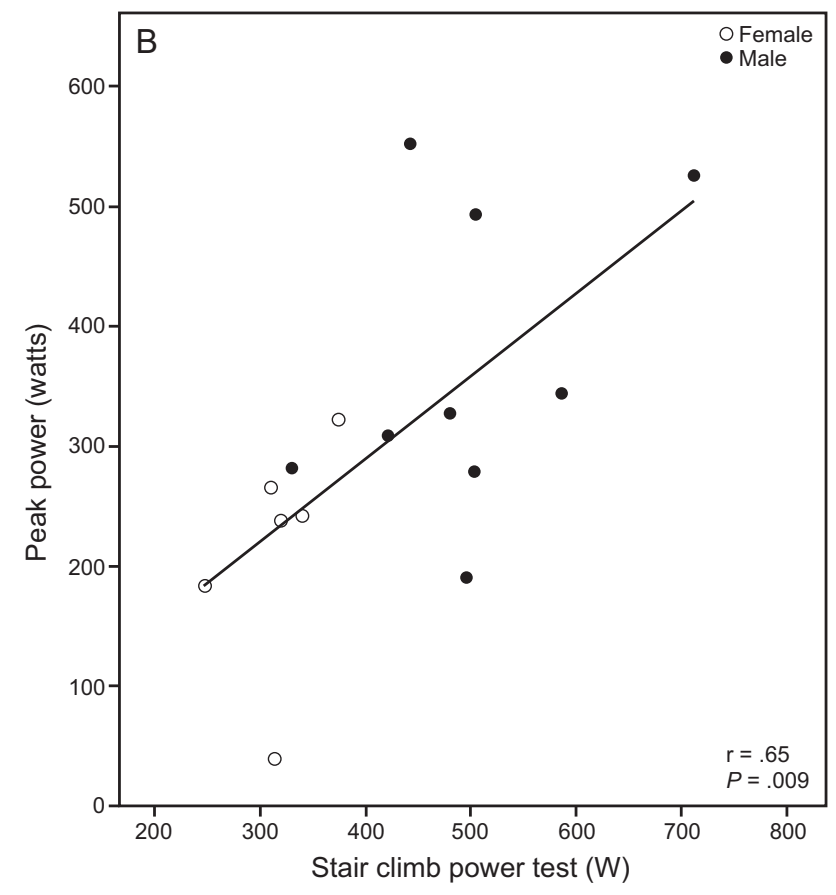

comparison with the computerized dynamometry. All functional tests were associated with quadriceps peak torque from the Biodex dynamometer, with the strongest correlation with the stair-climb power test. The vertical jump, triple hop distance, and stair-climb power tests are tasks that require lower-extremity power and, accordingly, showed moderate relationships to quadriceps peak power and velocity as measured by using dynamometry. Our 


\section{Muscle Strength and Power in Adults With CF}

results demonstrated that functional tests were valid measures of quadriceps strength and power in adults with cystic fibrosis. Furthermore, these tests are more easily implemented in the clinical setting because they require minimal space and equipment, and do not cause any adverse events.

The 30-s sit-to-stand test correlated with knee extensor peak torque (strength) but not with peak power or velocity in adults with cystic fibrosis. Previous studies in older adults $^{21}$ and people with COPD ${ }^{22}$ showed similar relationships. Gruet et al23 used the 1-min sit-to-stand test in adults with cystic fibrosis and found that it was correlated to peak aerobic capacity but not quadriceps strength. This may be due to the longer duration of the 1-min sit-to-stand test, which tasks the aerobic metabolism of the muscle more than strength. The sit-to-stand test, however, may have a ceiling effect in younger adults because it was developed for an older adult population. The subjects in our study completed 24 repetitions on average, with some individuals completing $30-31$ repetitions. This was considerably higher than normative values in older adults, which are typically between 12 and 16 repetitions. ${ }^{18}$

Muscle power is gaining attention as an important predictor of disability in older adults and may actually decline earlier with aging than peak muscle strength. ${ }^{24}$ Muscle power has not previously been evaluated in adults with cystic fibrosis but may also be a sensitive marker of changes in muscle function over time or after a period of hospitalization. The vertical jump, triple hop distance, and stairclimb power tests are tasks that require lower-extremity muscle power and, accordingly, showed moderate relationships to quadriceps peak power in our study. The vertical jump test has been used in cystic fibrosis, with one study that showed reduction compared with controls ${ }^{25}$ and the other study that showed no difference. ${ }^{26}$ In our study, the stair-climb power test was the best correlate of strength and power in cystic fibrosis and is a challenging test that could be applied across a spectrum of ages and functional levels.

There were limitations to the current study, which should be considered in the interpretation of the results. The sample size was small and only included individuals with mildto-moderate lung disease $\left(\mathrm{FEV}_{1} \%\right.$ predicted range, 39109 ), and the majority of subjects were $<30$ y old. A larger study, which included a wider spectrum of disease severity, stratified by age and sex, is needed to understand whether the functional tests are representative of muscle strength and power in all adults with cystic fibrosis. We conducted muscle power testing by using the isotonic function of the Biodex dynamometer, which has a preset external load that is moved throughout the range of motion. This method has previously been used;17 however, there are other methods for evaluating muscle power by using isometric and isokinetic modes. ${ }^{27}$ Our study was limited to the quadriceps muscle; however, sit-to-stand, jumping, and stair climbing also require other lower-extremity muscles (eg, gluteals and plantar flexors). The function of these other muscle groups should also be explored in adults with cystic fibrosis. Furthermore, the stair-climb power test requires access to a 10-step staircase, which may not be available in all clinical settings. This is an important consideration in the translation of these research findings into the clinical setting and may impact the uptake of the stairclimb power test by health-care professionals.

\section{Conclusions}

We recommend that functional tests, particularly the stairclimb power test, be used in the clinical setting to assess quadriceps muscle strength and power. Future studies should examine the sensitivity of these tests in detecting change over time and the relationship of muscle function to clinically relevant outcomes in cystic fibrosis.

\section{REFERENCES}

1. Salvatore D, Buzzetti R, Baldo E, Forneris MP, Lucidi V, Manunza $\mathrm{D}$, et al. An overview of international literature from cystic fibrosis registries. Part 3. Disease incidence, genotype/phenotype correlation, microbiology, pregnancy, clinical complications, lung transplantation, and miscellanea J Cyst Fibros 2011;10(2):71-85.

2. Salvatore D, Buzzetti R, Mastella G. Update of literature from cystic fibrosis registries 2012-2015. Part 6: Epidemiology, nutrition and complications. Pediatr Pulmonol 2017;52(3):390-398.

3. Gruet M, Troosters T, Verges S. Peripheral muscle abnormalities in cystic fibrosis: Etiology, clinical implications and response to therapeutic interventions. J Cyst Fibros 2017;16(5):538-552.

4. Elkin SL, Williams L, Moore M, Hodson ME, Rutherford OM. Relationship of skeletal muscle mass, muscle strength and bone mineral density in adults with cystic fibrosis. Clin Sci (Lond) 2000; 99(4):309-314.

5. Pinet C, Cassart M, Scillia P, Lamotte M, Knoop C, Casimir G, et al. Function and bulk of respiratory and limb muscles in patients with cystic fibrosis. Am J Respir Crit Care Med 2003;168(8):989-994.

6. Troosters T, Langer D, Vrijsen B, Segers J, Wouters K, Janssens W, et al. Skeletal muscle weakness, exercise tolerance and physical activity in adults with cystic fibrosis. Eur Respir J 2009;33(1):99106.

7. Wieboldt J, Atallah L, Kelly JL, Shrikrishna D, Gyi KM, Lo B, et al. Effect of acute exacerbations on skeletal muscle strength and physical activity in cystic fibrosis. J Cyst Fibros 2012;11(3):209-215.

8. Martin C, Chapron J, Hubert D, Kanaan R, Honoré I, Paillasseur JL, al. Prognostic value of six minute walk test in cystic fibrosis adults. Respir Med 2013;107(12):1881-1887.

9. Robles PG, Mathur S, Janaudis-Fereira T, Dolmage TE, Goldstein RS, Brooks D. Measurement of peripheral muscle strength in individuals with chronic obstructive pulmonary disease: a systematic review. J Cardiopulm Rehabil Prev 2011;31(1):11-24.

10. Vaidya T, Chambellan A, de Bisschop C. Sit-to-stand tests for COPD: A literature review. Respir Med 2017;128:70-77.

11. Johnston KN, Potter AJ, Phillips A. Measurement Properties of Short Lower Extremity Functional Exercise Tests in People With Chronic Obstructive Pulmonary Disease: Systematic Review. Phys Ther 2017; 97(9):926-943. 


\section{Muscle Strength and Power in Adults With CF}

12. Bean JF, Kiely DK, LaRose S, Alian J, Frontera WR. Is stair climb power a clinically relevant measure of leg power impairments in at-risk older adults? Arch Phys Med Rehabil 2007;88(5):604-609.

13. Roig M, Eng JJ, MacIntyre DL, Road JD, Reid WD. Associations of the Stair climb power test with muscle strength and functional performance in people with chronic obstructive pulmonary disease: a cross-sectional study. Phys Ther 2010;90(12):1774-1782.

14. Harman EA, Rosenstein MT, Frykman PN, Rosenstein RM. The effects of arms and countermovement on vertical jumping. Med Sci Sports Exerc 1990;22(6):825-833.

15. Quanjer PH, Stanojevic S, Cole TJ, Baur X, Hall GL, Culver BH, et al. ERS Global Lung Function Initiative. Multi-ethnic reference values for spirometry for the 3-95-yr age range: the global lung function 2012 equations. Eur Respir J 2012;40(6):1324-1343.

16. Mathur S, Makrides L, Hernandez P. Test-retest reliability of isometric and isokinetic torque in patients with chronic obstructive pulmonary disease. Physiother Can 2004;56:94-101.

17. Accettura AJ, Brenneman EC, Stratford PW, Maly MR. Knee extensor power relates to mobility performance in people with knee osteoarthritis: cross-sectional analysis. Phys Ther 2015;95(7):989-995.

18. Jones CJ, Rikli RE, Beam WC. A 30-s chair-stand test as a measure of lower body strength in community-residing older adults. Res Q Exerc Sport 1999;70(2):113-119.

19. Hamilton RT, Shultz SJ, Schmitz RJ, Perrin DH. Triple-hop distance as a valid predictor of lower limb strength and power. J Athl Train 2008;43(2):144-151.

20. Munro B, Page E, Visintainer M. Statistical Methods for Health Care Research. 5th ed. Philadelphia: Lippincott, Williams \& Wilkins, 2005; 240 .
21. McCarthy EK, Horvat MA, Holtsberg PA, Wisenbaker JM. Repeated chair stands as a measure of lower limb strength in sexagenarian women. J Gerontol A Biol Sci Med Sci 2004;59(11):1207-1212.

22. Butcher SJ, Pikaluk BJ, Chura RL, Walkner MJ, Farthing JP, Marciniuk DD. Associations between isokinetic muscle strength, highlevel functional performance, and physiological parameters in patients with chronic obstructive pulmonary disease. Int J Chron Obstruct Pulmon Dis 2012;7:537-542.

23. Gruet M, Peyré-Tartaruga LA, Mely L, Vallier JM. The 1-minute sit-to-stand test in adults with cystic fibrosis: correlations with cardiopulmonary exercise test, 6-minute walk test, and quadriceps strength. Respir Care 2016;61(12):1620-1628.

24. Reid KF, Pasha E, Doros G, Clark DJ, Patten C, Phillips EM, et al. Longitudinal decline of lower extremity muscle power in healthy and mobility-limited older adults: influence of muscle mass, strength, composition, neuromuscular activation and single fiber contractile properties. Eur J Appl Physiol 2014;114(1):29-39.

25. Arikan H, Yatar I, Calik-Kutukcu E, Aribas Z, Saglam M, VardarYagil N, et al. A comparison of respiratory and peripheral muscle strength, functional exercise capacity, activities of daily living and physical fitness in patients with cystic fibrosis and healthy subjects. Res Dev Disabil 45-46:147-156.

26. Sahlberg ME, Svantesson U, Thomas EM, Strandvik B. Muscular strength and function in patients with cystic fibrosis. Chest 2005; 127(5):1587-1592.

27. Webber SC, Porter MM. Reliability of ankle isometric, isotonic, and isokinetic strength and power testing in older women. Phys Ther 2010;90(8):1165-1175. 